\title{
Fractional Frequency Synthesizers With Low Order Time-Variant Digital Sigma-Delta Modulator
}

\author{
Victor Rodolfo Gonzalez-Diaz, Member, IEEE, Aldo Pena-Perez, Member, IEEE, and \\ Franco Maloberti, Fellow, IEEE
}

\begin{abstract}
This paper proposes the use of a digital $\Sigma \Delta$ modulator with pseudorandom variation of coefficients. The method improves PLL-based Fractional Frequency Synthesizer's performance while using a low order digital $\Sigma \Delta$ modulator. The time variant coefficients, in a low order digital $\Sigma \Delta$ modulator, significantly enlarge the pseudorandom output pattern period thus avoiding critical spur tones in the fractional synthesizer's phase-noise. Behavioral simulations let us verify that the proposed technique operates better than a higher order modulator with additive dither. With a low order $\Sigma \Delta$ architecture, the programmable divider phase-selection logic and frequency range of operation parameters can be relaxed.
\end{abstract}

Index Terms - Digital, fractional frequency synthesizers, MASH, phase noise, Sigma-Delta, spur tones, time variant.

\section{INTRODUCTION}

F RACTIONAL frequency synthesizers are common blocks in wireless communication systems and clock generators [1]. As known, they are made by the loop of a phase-to-frequency detector $(P F D)$, a charge-pump $(C P)$, a low pass filter $(L P F)$, a voltage controlled oscillator $(V C O)$ and a multimodulus integer divider, as shown in Fig. 1. A digital $\Sigma \Delta$ modulator, with constant input $(X)$, pseudorandomly determines the variable division factor $(N,(N+1), \ldots)$. The ratio between the $\Sigma \Delta$ input $X$ and its full scale $2^{m}$ controls the output frequency $f_{\text {out }}=\left(N+\left(X / 2^{m}\right)\right) f_{\text {ref }}$.

The spectral content of the generated signal is limited by phase noise characteristics. In one hand, charge pump noise is of high concern for close frequencies from the carrier. On the other hand, the main noise source at medium distance from the carrier is the shaped quantization noise caused by the digital $\Sigma \Delta$ [2]. At very high frequencies the phase noise is dominated by the VCO's noise. Accordingly, a careful design optimizes performance near and at far frequencies offset from the carrier. A good choice could be a low order $\Sigma \Delta$ modulator capable to reduce shaping for intermediate frequencies, whereas increasing quantization noise for ranges where charge pump noise is dominant. Unfortunately, with constant inputs, the periodic characteristic of conventional low order $\Sigma \Delta$ produces spur tones and

Manuscript received September 03, 2011; revised December 23, 2011 and February 25, 2012; accepted March 08, 2012. Date of publication April 09, 2012; date of current version May 09, 2012. This paper was recommended by Associate Editor T. S. Lande.

V. R. Gonzalez-Diaz is with the Autonomous University of Puebla (BUAP), 72000 Puebla, Mexico (e-mail: vr.gonzalezdiaz@gmail.com).

A. Pena-Perez and F. Maloberti are with the Department of Electronics, Pavia University, 27100 Pavia, Italy.

Digital Object Identifier 10.1109/TCSI.2012.2191317

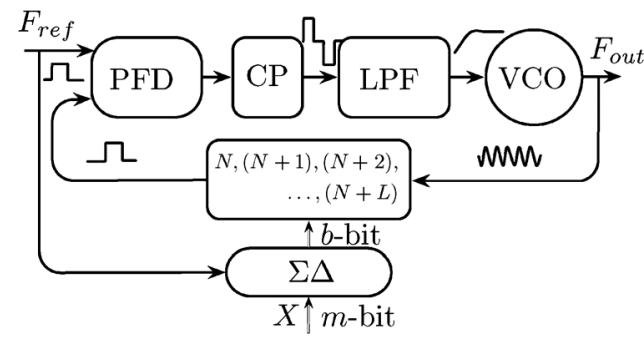

Fig. 1. Block diagram of fractional synthesizer.

this (obviously unwanted) restricts designers to use high order schemes. They lead to a noise shaping figure that is higher than required but are less affected by spur tones.

The charge pump and the VCO experience nonlinearity. Thus, the tones generated by the digital $\Sigma \Delta$ modulator have the probability to produce modulation products that possibly fall close to the carrier. Reference [3] shows that $\Sigma \Delta$ periodicities and charge pump nonlinearities typically create tones in the frequency range where the charge pump noise dominates.

The use of high order modulators limit spur tones but creates problems to modulator's stability especially when the scheme includes loops of multiple accumulators. Otherwise they must be MultistAge-noise-SHaping (MASH) [4] architectures that, both, lead to multiple quantization levels. Accordingly, the programable divider changes in the range $N \ldots(N+L)$ (being $L=2^{b}$ and $b$ the number of $\Sigma \Delta$ output bits, see Fig. 1). For instance, a third order $\Sigma \Delta \mathrm{MASH}$ has a 3-bit output sequence which means a programmable divider with 8 levels. A second $\operatorname{order} \Sigma \Delta \mathrm{MASH}$, that leads to a 2-bit output sequence, requires a 4-level programmable divider. Obviously, moving from 3 to 2 bit simplifies most of the programmable divider topologies and improve reliability (as the phase selection logic and multiplexor depend on timing constraints imposed by the number of levels) [5], [6]. Thus the optimal solution is the lowest order in the $\Sigma \Delta$ modulator having a non easily detectable periodic behavior. The approaches used in [7]-[11] modify the digital accumulator as to obtain a prime modulus quantization. The benefits cost not only additional hardware but also a post filter stage that restricts the use of this architectures in a fractional synthesizer designed for data modulation and up conversion [12]. Particularly, tones are significant for digital $\Sigma \Delta$ modulators with order lower than three. The use of digital dither added to input or to key points of the architecture [13], [14] also reduces tones appearance but the method is not effective for low order topologies.

This paper achieves a significant randomization of the $\Sigma \Delta$ output by dithering its coefficients with multiplicative factors 


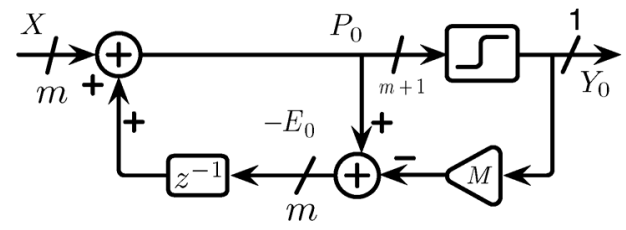

Fig. 2. Block diagram of digital accumulator.

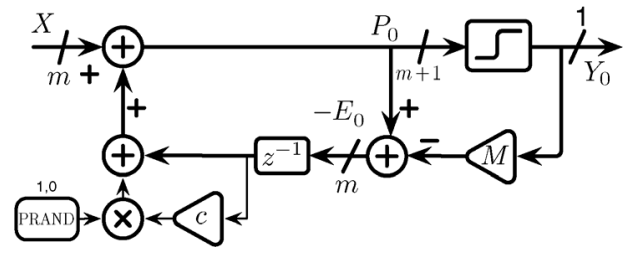

Fig. 3. Time variant accumulator.

rather than simply adding dithered terms. The method, as discussed shortly, is very effective even for low order architectures. The proposed scheme and theoretical aspects are illustrated in Section II. Section III validates the approach with behavioral simulations. Finally, Section IV presents the conclusions of this work.

\section{TIME VARIANT $\Sigma \Delta$ MODULATOR}

For an effective fractional division of the VCO frequency, it is desired the integer division moduli to change around the desired value according to a random process. In addition, the power spectrum of the process must contribute with negligible noise at zero distance from the desired value. This corresponds to frequency-shaped random processes. A digital $\Sigma \Delta$ modulator with dithering is a suitable alternative to obtain the result. However, additive dithering is not particularly effective because addition is a linear operation. An alternative solution is to use multiplicative dithering. As shown below, it turns to be much more effective because multiplication is a nonlinear operation for digital MASH modulators that helps on breaking periodicity.

\section{A. $\Sigma \Delta$ Time Variation Coefficients}

Let us consider the simplest digital $\Sigma \Delta$, the first order scheme of Fig. 2. It corresponds or is closely related to the digital implementation. The addition represents an m-bit digital accumulator whose output is $e_{0}$.

The quantization error $e_{0}$ at the clock index $i$ is a discrete time $m$-bit sequence given by

$$
-e_{0}[i]=\left(x[i]-e_{0}[i-1]\right) \bmod M .
$$

That is useful to estimate the periodicity of the quantization error. If $e_{0}[i]$ has a period $I$ then $e_{0}[i]=e_{0}[i+I]$ and it is possible to achieve the expression

$$
(I x) \bmod M=0 .
$$

Equation (2) determines the period $I$ of the quantization error $e_{0}$ [8]. It depends on the input value $x$ and increases with the quantization modulus $M$. It can be maximized by making the $k M / x$ ratio non integer for any integer value of $k \in[1, \infty]$. If the period of the sequence length becomes very large, the quantization error can be treated as an additive white noise signal. Under these conditions it is possible to analyze the accumulator of Fig. 2 in the $z$ domain. Signals $P_{0}(z)$ and $Y_{0}(z)$ are described by

$$
\begin{aligned}
& P_{0}(z)=X(z)-M E_{0}(z) z^{-1} \\
& Y_{0}(z)=\frac{1}{M}\left[X(z)+\left(1-z^{-1}\right) E_{0}(z)\right]
\end{aligned}
$$

where the full scale of $X$ is set to $M$.

By inspection of Fig. 2, the signal transfer function (STF) is equal to 1 and the noise transfer function (NTF) given by

$$
N T F(z)=\left(1-z^{-1}\right)=\left(1-b_{1} z^{-1}\right)
$$

where $b_{1}$ (here equal to 1 ) is used to generalize the coefficient of the NTF. The value of $b=1$ sets the zero at $z=1$ in the $z$ plane. Changing $b_{1}$ in a pseudorandom manner around the expected value 1 not only blurs the noise transfer function but also limits to periodicity. Strictly speaking, time variant coefficients do not allow to use the $z$ variable anymore. Here we are using the $z$-domain analysis with similar approximations to those considered for analog $\Sigma \Delta$ modulators [15]. If $b_{1}=\left(1-c_{1}\right)$ the noise transfer function becomes

$$
N T F(z)_{o n e}=\left[1-\left(1-c_{1}\right) z^{-1}\right] ;
$$

i.e., the zero slightly moves on the real axis inside the unity circle. If the mean value of $c_{1}$ is small, and its rate of change is large enough, the time variant operation does not affect stability. Moving the NTF zero from $z=1$ limits the noise shaping near $f=0$. The noise spectrum, therefore, shows a noise floor that increases with $c_{1}$ like it happens when an equivalent random noise (or dither) is added to input. Note that this increase for quantization noise floor will not affect to fractional synthesizer's phase noise as it is raised for low frequencies where the charge pump noise is more significative. This aspect will be pointed in detail with behavioral simulation results in the following sections.

Fig. 3 shows a possible implementation of the time variant first order $\Sigma \Delta$ modulator. An auxiliary pseudorandom bit stream multiplies the coefficient $c=c_{1} / 2$ by $(1,0)$, yielding as a result, the pseudorandom variation of the $z^{-1}$ multiplier. The scheme requires an additional multiplier and an extra adder to combine the time variant operation with the delayed version of $E_{0}$.

Fig. 4 shows an alternative solution that does not use a multiplier. The quantization error, passed through a 1 st order digital $\Sigma \Delta$, generates a single bit output that multiplied by a pseudorandom $(1,0)$ coefficient enables the addition of $c$. Notice that the scheme does not use $c E_{0}$ because at the output of the auxiliary $\Sigma \Delta$ there is $E_{0}$ plus an extra quantization error. This is not a counteraction; instead, it renders assistance to break periodicity. Moreover, the multiplication requires only a logic AND operation and a simple linear feedback shift register (LFSR) suitably generates the 1-bit pseudorandom signal to vary the coefficient.

\section{B. Extension to $\Sigma \Delta$ Time Variant MASH Scheme}

As a first approach, all coefficients should be changed within time to break the periodicity in a digital MASH. For a generic $n$-order TV MASH architecture, like the one shown in Fig. 5, 


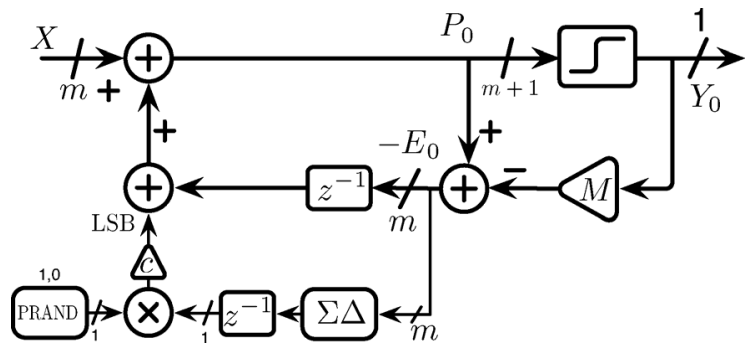

Fig. 4. Effective coefficient variation.

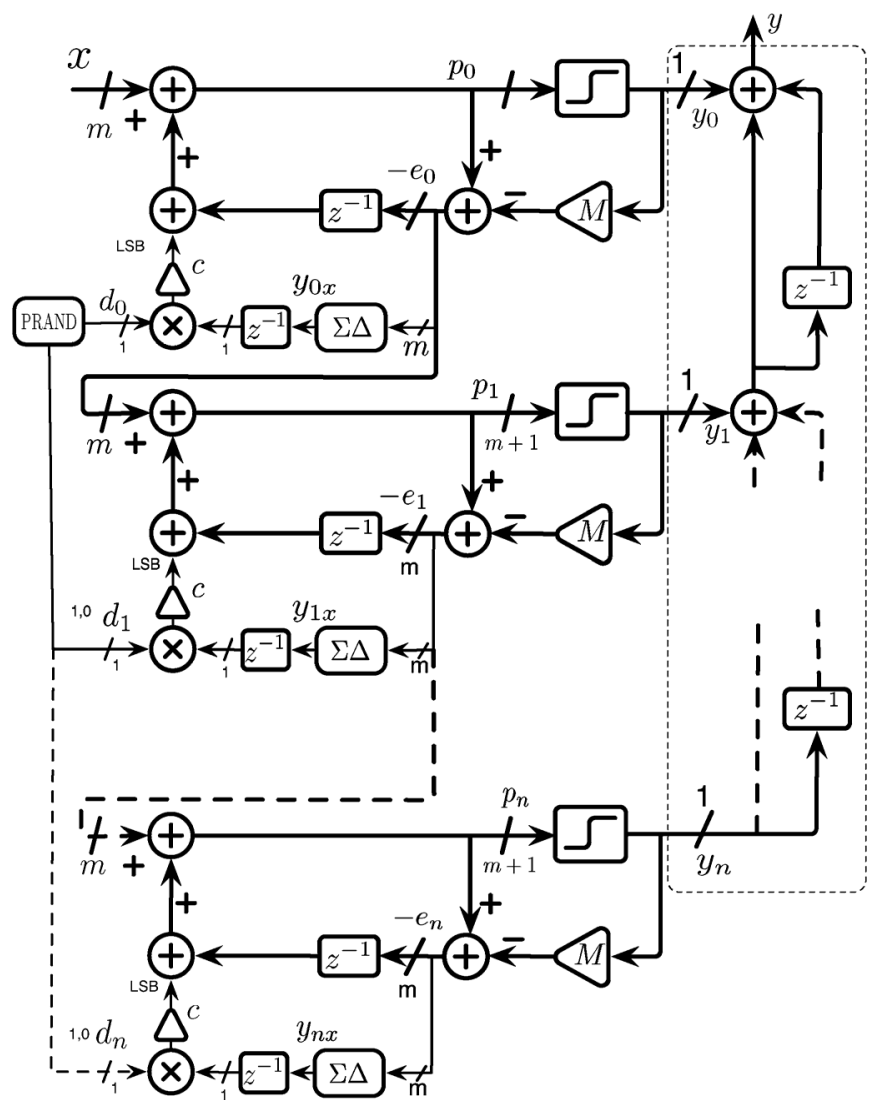

Fig. 5. Generic implementation for an $n$-th order time variant MASH $\Sigma \Delta$ modulator.

$2 n$ digital accumulators would be needed. By inspection of the figure the single-bit output for the $n$-th stage is

$$
\begin{aligned}
Y_{n}=-E_{n-1} & +\left(1-z^{-1}\right) E_{n} \\
& +c z^{-1}\left[\left(1-z^{-1}\right) E_{n x}-E_{n}\right] \quad \text { for } n>0
\end{aligned}
$$

where $\mathrm{c}$ is the scaling to a LSB for the time variant coefficient and $E_{n x}$ is the quantization error for the additional $\Sigma \Delta$ stage (with output $Y_{n x}=-E_{n}+\left(1-z^{-1}\right) E_{n x}$ ). The latter converts the quantization error to a 1-bit sequence as to make the one-bit product with $d_{n}$. Notice that (7) is the same noise shaping for a plain MASH structure but with an additional term which affects shaping for low frequency ranges with a factor that is not significant for close-in phase noise in fractional synthesizers.

However, implementing the TV approach in this form (as shown in Fig. 5) may lead to an undesirable increase of hardware complexity when the MASH architecture has more than

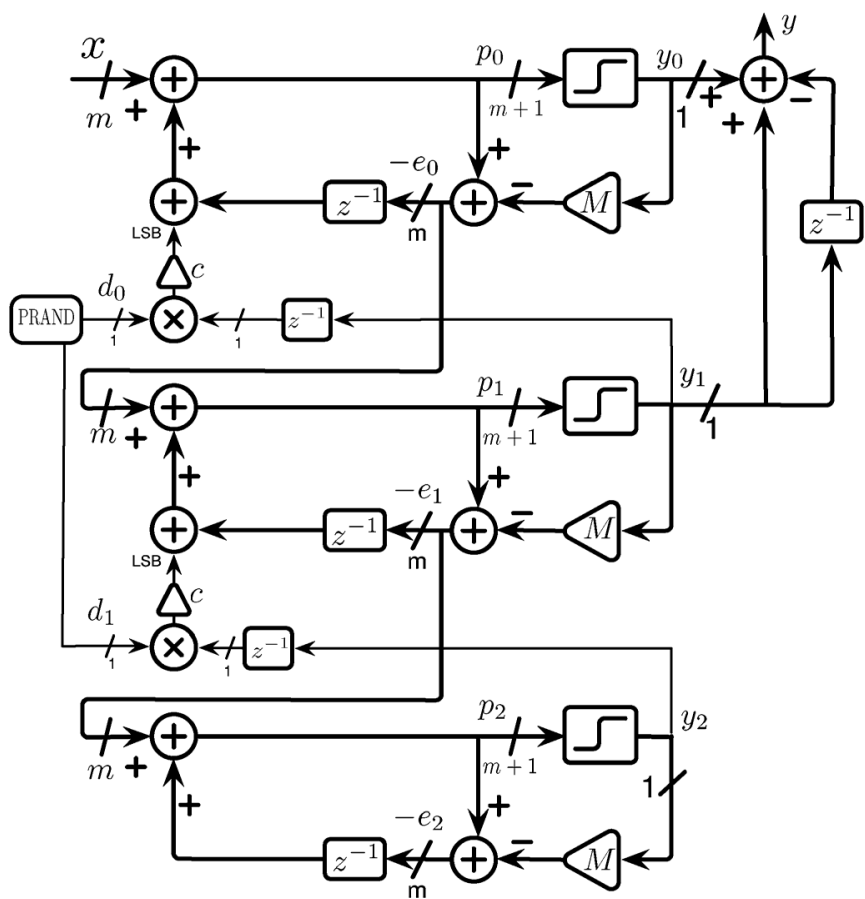

Fig. 6. Time variant MASH 1-1 $\Sigma \Delta$ implementation.

one stage. Nonetheless, a MASH topology limits the cost because each stage accumulates the quantization error of the previous stage. Thus, the output of each stage, multiplied by $c_{1}$, is useful for the time variant injection in the previous stage. This attribute yields, as a particular case, the second order TV MASH scheme of Fig. 6. In general, for an $n$-th order topology with the arrangement of Fig. 6 the single-bit output sequence for the $n$-th stage is given by

$$
\begin{aligned}
& Y_{n}=-E_{n-1}+\left(1-z^{-1}\right) E_{n} \\
& +c z^{-1}\left[\left(1-z^{-1}\right) E_{n+1}-E_{n}\right] \text { for } n>0
\end{aligned}
$$

which is practically the same noise shaping than the implemented in Fig. 5. For a $n$ stages TV MASH architecture only $(n+1)$ accumulators are required.

Since it is important to study the influence of time variant coefficients on noise shaping, we consider the case of a TV MASH 1-1 topology to explore the behavior of low-order schemes. The overall 2-bit output sequence after noise cancellation logic in Fig. 6 is

$$
Y=X+\left(1-z^{-1}\right)^{2} E_{1}+c z^{-1}\left[\left(1-z^{-1}\right)^{2} E_{2}-E_{0}\right]
$$

and for an $n$-order TV MASH the output sequence is

$$
Y=X+\left(1-z^{-1}\right)^{n} E_{n-1}+c z^{-1}\left[\left(1-z^{-1}\right)^{n} E_{n}-E_{0}\right] .
$$

Fig. 7 compares the noise transfer characteristic for a second and third order TV MASH schemes with plain structures. The limit in all cases is the quantization error from the first accumulator $E_{0}$ whose coefficient is not shaped but the contribution is for noise levels bellow the LSB. This shows that, when used 


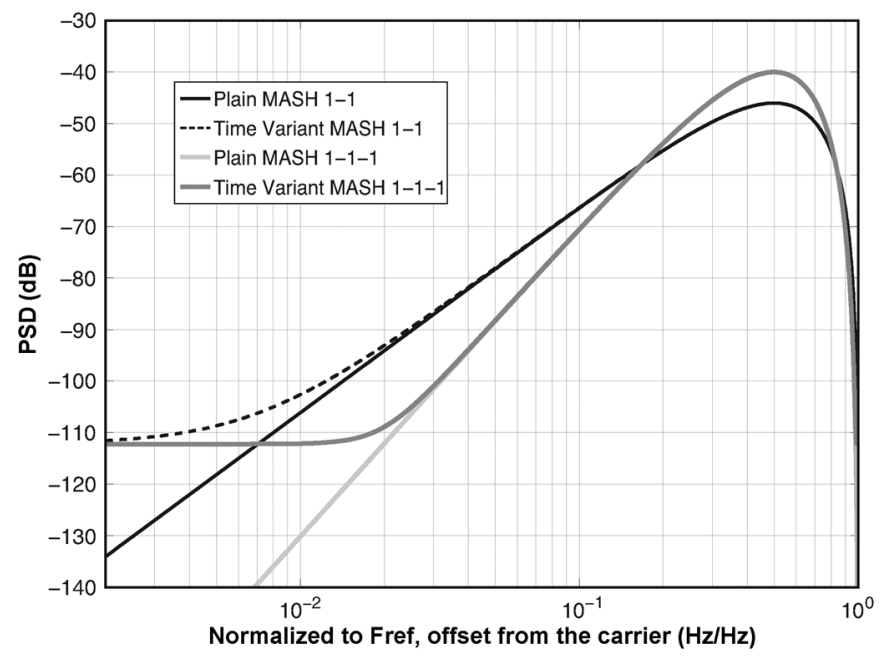

Fig. 7. Estimated noise shaping from TV MASH compared to plain MASH schemes.

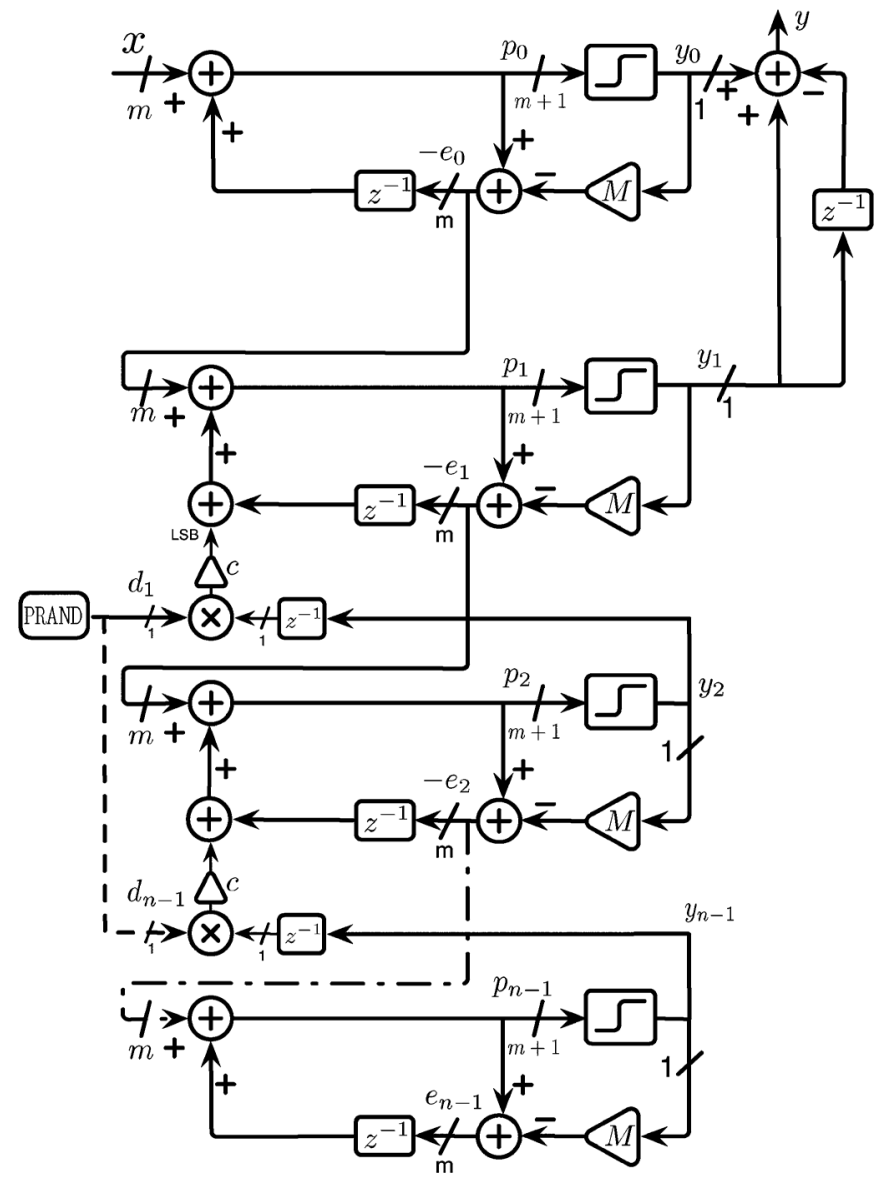

Fig. 8. MASH 1-1 with time-variant second stage (the last coefficient is subsequently changed to reduce correlation between quantization noises).

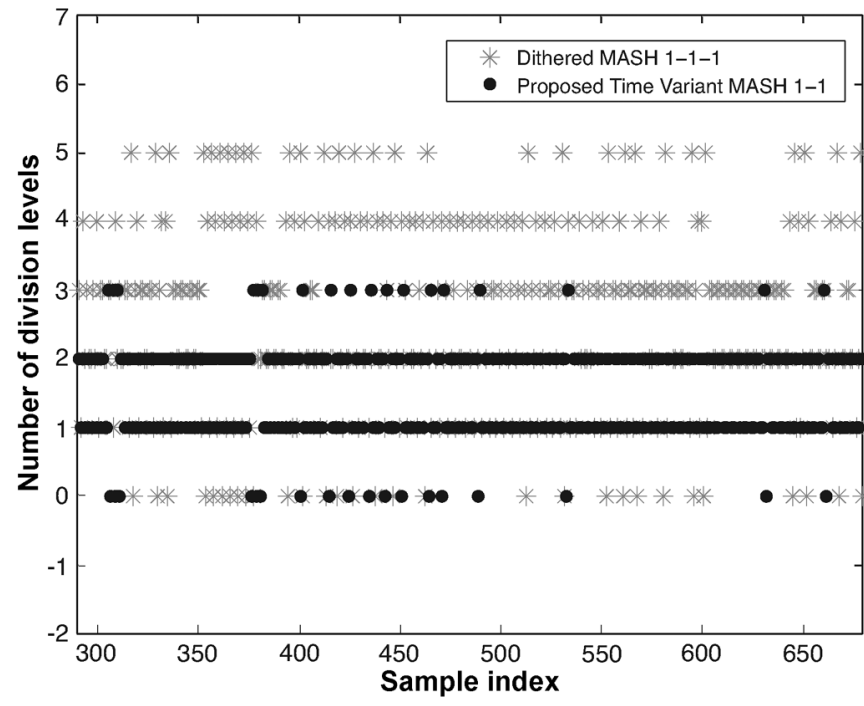

Fig. 9. Division levels in the fractional synthesizer for a traditional dithered MASH 1-1-1 and the time variant MASH 1-1.

in fractional synthesizers, will not impact negatively on phase noise.

\section{Periodicity of a Second Order Time Variant MASH $\Sigma \Delta$}

As mentioned before, the TV MASH 1-1 uses a LFSR as an auxiliary pseudorandom number generator $(P R N G)$ to change within time the products with the feedback quantization errors. So, to increase the possibilities for uncorrelated pseudorandom variation all coefficients are changed with a different output from the auxiliary PRNG (see Fig. 6). To estimate the periodicity of the quantization error of time-variant $\Sigma \Delta$ modulator is difficult because it involves the solution of a nonlinear system of modular arithmetic equations. Here, we just define the problem and expose some intuitive considerations.

From the analytic point of view it is necessary to study the time evolution of the scheme. Consider, for example, the architecture of Fig. 6 . Assuming that $\left[x, \bar{e}_{0}, \bar{e}_{1}, \bar{e}_{2}\right]$ is the initial state of the system, the quantization errors of first and second stage are represented by the equation at the bottom of the page.

Where the Pochhammer symbol $i^{(k)}$ is used for rising sequential products $i^{(k)}=i(i+1)(i+2) \ldots(i+k-1)$. Note the $\mathrm{c}$ symbol does not appear on (11) as the feedback operations are on the carry inputs for every accumulator, therefore, normalizing the products leads to the sequence of terms in (11).

To obtain the period $I$ it is necessary to solve the above set of (11) for the case the quantization vector $e_{k}[i+I]=e_{k}[i]$ which means $e_{k}[I]=\bar{e}_{k}$. Note that solving this third order nonlinear congruential system requires to look for the congruential vector 


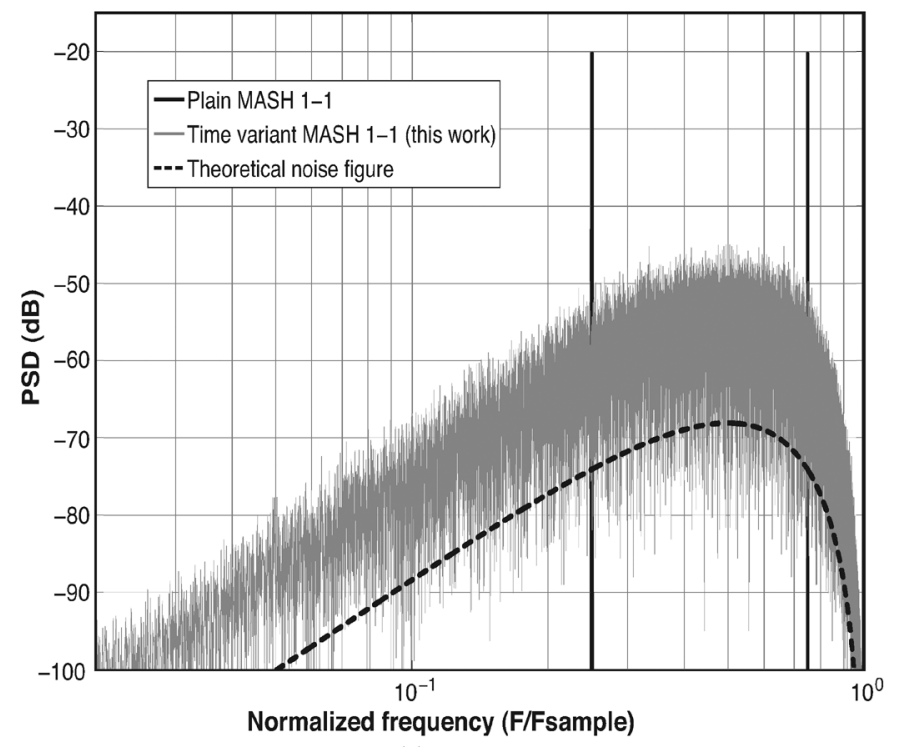

(a)

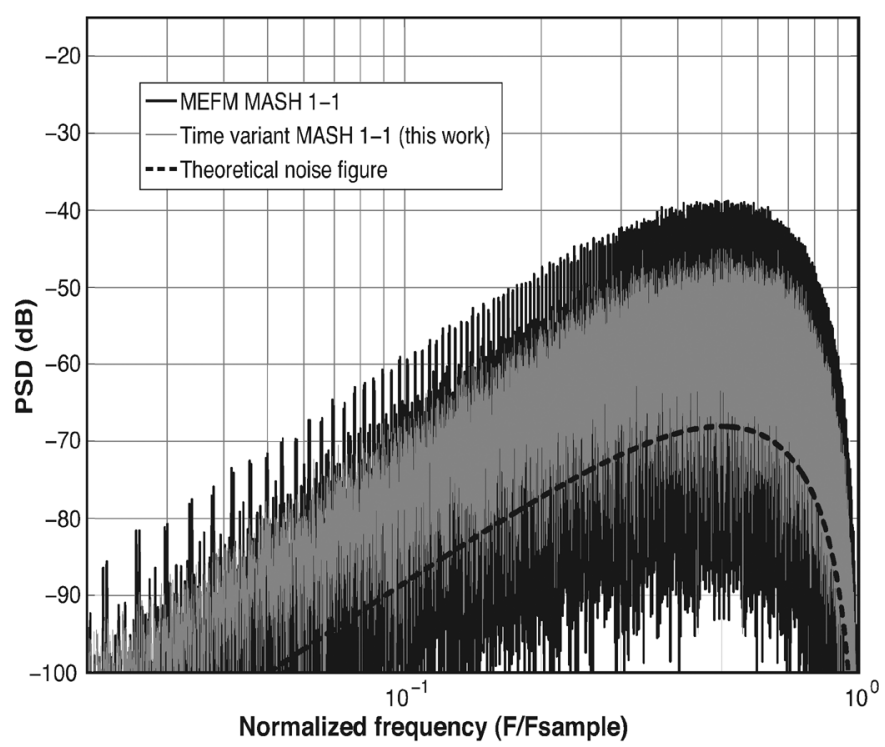

(b)

Fig. 10. Power spectral density of time variant MASH 1-1 compared to other second order topologies. (a) Comparison between plain MASH 1-1 and TV MASH 1-1. (b) Comparison between plain MEFM 1-1 and TV MASH 1-1.

solving the system for $2^{(4) m}$ different initial conditions, where $m=2^{b}$ being $b$ the number of bit of the accumulators.

An intuitive explanation of the effectiveness of the time variant method is the following: a pseudorandom sequence has the same numbers of ones and zeros if generated by a maximum length LFSR [16]. Therefore, the accumulation over the repetition period of the pseudorandom sequence is zero. The multiplication of the pseudorandom sequence by the quantization error produces a sequence whose periodicity depends on both the periodicity of the pseudorandom and the quantization error, leading to a significative improvement compared to a simple dithered solution considering the same LFSR size (for the studied case in this paper the PRNG is an $m$-bit LFSR).

\section{Time-Variant MASH Scheme: Benefits and Trade-Offs}

The scheme of Fig. 6 is the cascade of two time variant first order digital $\Sigma \Delta$. The overall effectiveness is excellent but the shift of the NTF zeros from the $z=1$ position rises the noise floor for both stages [as predicted by (9)]. The NTF of the second stage is referred to the input traversing through the NTF of the first stage. Therefore, to avoid the rise of the noise floor only the coefficient in the last stage should be varied within time (in this case the NTF of the third stage is not refereed to a subsequent and shaping is maintained). For this scenario, according to the evolution of the system as stated by (11), the only term with non linearity would be the $e_{2}[i]$ sequence and periodicity will be restricted by the original pseudorandom generator (in our case is a simple $m$-bit LFSR).

Alternatively, coefficients for cascade accumulators (not introduced to the noise cancellation logic) could be constantly varied in a chain of nested time variant coefficients as shown in Fig. 8. However, the outputs that contribute to the overall input are the first two terms $y_{0}, y_{1}$ and adding nested accumulators slightly improves the periodicity of the overall output. For the shake of brevity we conclude that, for fractional synthesizers aspects, the noise shaping raise due to varying the first coefficient yields better results. Moreover, as will be discussed in next section, it is not of big impact on the overall phase noise figure.

Note the time variant MASH modulator has an unitary signal transfer function $(S T F)$, see (9). This means that the $\Sigma \Delta$ input is not affected by the time variation coefficients, albeit the latter enlarges the periodicity of the output sequence $y[n]$ on Fig. 6 . This is beneficial for applications where the frequency output accuracy is of big concern and the fractional division should not have a variation. Having a STF different than unity makes necessary another post filter stage at the $\Sigma \Delta$ output [9].

Besides, if the fractional synthesizer is to be used in data modulation and up conversion, the $\Sigma \Delta$ modulator STF must be unitary to allow the data modulation at the input $x$. This is a principal characteristic of fractional frequency synthesizer based transmitters in which modulation block is the input to the $\Sigma \Delta$ modulator that controls to the programmable divider [12]. So, the time variant MASH proposed in this work is compatible with these type of transmitters with the advantage of spur noise suppression.

Another benefit of a low order topology in $\Sigma \Delta$ fractional frequency synthesizers is, however, the smoother noise shaping for high frequencies offset from the carrier and the reduction of the number of levels to obtain the fractional division. Fig. 9 shows that for the proposed second order time variant the number of levels needed for fractional division is only 4 , which means 2-bit in the programmable divider. This feature relaxes the constraints for the programmable divider. The principal issue with a wide range programable divider is the probability of glitches within the phase-selector logic and some times it is necessary to add an inherently glitch free technique as proposed in [17]. Moreover, in the design of phase-switching prescalers the operation speed is not limited by the first stage prescalers. The delay in the multiplexer and control logic are of major concern and, for high-speed operation, a long time windows is desired 
TABLE I

Hardware Comparison of Spur Tones Reduction Techniques for Digital $\Sigma \Delta$ in Fractional Frequency Synthesizers

\begin{tabular}{|c||c||c||c||c||c||c|}
\hline Architecture & $\begin{array}{c}\text { Adders } \\
\text { of }(m \text {-bit) }\end{array}$ & $\begin{array}{c}\text { Registers } \\
\text { of }(m \text {-bit })\end{array}$ & $\begin{array}{c}\text { Output } \\
\text { Filter }\end{array}$ & $\begin{array}{c}\text { Input } \\
\text { dependence }\end{array}$ & Tones & $\begin{array}{c}\text { Div. levels } \\
\text { in Freq. Synt. }\end{array}$ \\
\hline \hline Plain MASH 1-1-1 & 3 & 3 & no & no & yes & 8 \\
MEFM MASH 1-1-1 [9] & 7 & 5 & yes & no & yes (envelope) & 8 \\
Feedback dithering MASH 1-1-1 [18] & 6 & 5 & yes & yes & yes & 8 \\
Plain MASH 1-1 & 2 & 2 & no & no & yes & 4 \\
MEFM MASH 1-1 [9] & 7 & 3 & yes & no & yes (envelope) & 4 \\
T. V. MASH 1-1 (proposed) & 3 & 4 & no & no & no & 4 \\
\hline
\end{tabular}

for phase switching. In a phase-selection technique with forward-switching, the frequency range of operation decreases as $\mathrm{N}$ increases [5].

\section{VALidATion of THE Method With BehaVioraL SIMULATIONS}

According to last section, we expect the time variant $\Sigma \Delta$ modulator to reduce tones from the output spectrum in fractional synthesizers. This section presents simulation results that verify the effectiveness of the method respect to already proposed techniques. For the simulation results we considered an 8-bit resolution for all digital $\Sigma \Delta$ modulators.

\section{A. Digital $\Sigma \Delta$ Tests}

Conventional and time variant topologies were simulated using a $x=128$ constant input to achieve a fractional value of $128 / 256$. It ensures the test with the worst conditions as the periodicity is critical at this point, according to (11).

Fig. 10(a) shows the output spectrum comparison between the proposed time variant MASH 1-1 of Fig. 6 (with an 8-bit LFSR as the pseudorandom number generator) with a plain MASH 1-1. Fig. 10(b) presents the comparison with the state of the art second order modified error feedback modulator (MEFM) [7], [9]. Results show that, for frequencies relevant to fractional synthesizers, the time variant MASH 1-1 improves the power spectrum by about $-10 \mathrm{~dB}$ and $-30 \mathrm{~dB}$ compared to the MEFM 1-1 and the plain MASH 1-1, respectively. Much more important is the reduction of tones as made evident by large data sequences used for the fast Fourier transform (FFT), that grants a large processing gain $(-10 \log (N / 2))$ [15]. For computational trade-offs the results on Fig. 10 were obtained with a $2^{21}$ sequence size. The spectrum of higher order architectures obviously improves at low frequency but the time variant scheme generates smaller tones.

Table I compares various features of previous-art second and third order modulators with our proposed second order time variant MASH modulator. Results indicate that the second order time variant has a better trade-off compared to all counterparts, when used in frequency synthesizer loops. The MASH 1-1-1 needs 3 m-bit adders and 3 m-bit registers but presents a periodicity on the output sequence. The MEFM 1-1-1 and effective feedback dithering need 7 adders but still present tones. A plain MASH 1-1 uses less hardware but the presence of tones yields a worst behavior. The time variant MASH 1-1 requires three adders and four m-bit registers with two AND gates to vary, with a product fashion, the coefficients. The required

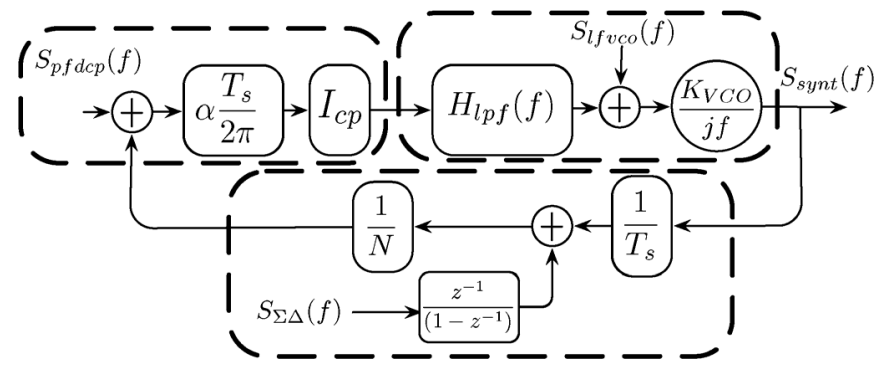

Fig. 11. Frequency domain model including important noise sources.

TABLE II

Simulated Fractional Synthesizer Parameters

\begin{tabular}{|c|c||c|c|}
\hline Parameter & Value & Parameter & Value \\
\hline \hline$K_{V C O}$ & $666 \frac{M H z}{V}$ & $I_{C P}$ & $10 \frac{\mu A}{V}$ \\
$\frac{x}{2^{m}}$ & $\frac{77}{256}$ & $\Delta I_{C P}$ & $\pm 20 \%$ \\
$S_{p f d c p}(f)$ & $1.6 x 10^{-24} \frac{A^{2}}{H z}$ & $S_{l f v c o}(f)$ & $2 x 10^{-22} \quad \frac{V^{2}}{H z}$ \\
$f_{\text {ref }}$ & $25 M H z$ & $f_{c}$ & $270 \quad k H z$ \\
LPF & 3 rd order & Division & $N=(64 . .72)$ \\
\hline
\end{tabular}

hardware corresponds to an affordable increase, compared to a plain third order architecture, and has a better trade-off for phase noise shaping and reduced presence of tones. Thus, a TV MASH 1-1 scheme enables a lower number of levels in the programmable divider, usually constructed by a multiplexer and control logic phase-selection techniques [19], [20]. For a phase-selection based programmable frequency divider, the propagation delay in the multiplexer and the control logic are or big concern for a good performance. Thus, for these type of phase-switching programmable dividers, the operating speed is not only limited by the prescaler but also for the time window the number of input periods that the control logic must use to change between levels. That is, the frequency covering range decreases as $\mathrm{N}$ increases. Relaxing these requirements increase the reliability on the programmable divider as the presence of glitches will be limited [5], [17].

On the other hand, the proposed TV MASH does not use filter stages, a side benefit when the frequency synthesizer operates as a digital data modulator and up conversion in wireless communication systems.

\section{B. Phase Noise Figure}

The estimated result of the expected phase noise figure from the TV MASH transference gives as a result a good trade-off between noise shaping and appearance of tones, compared to 


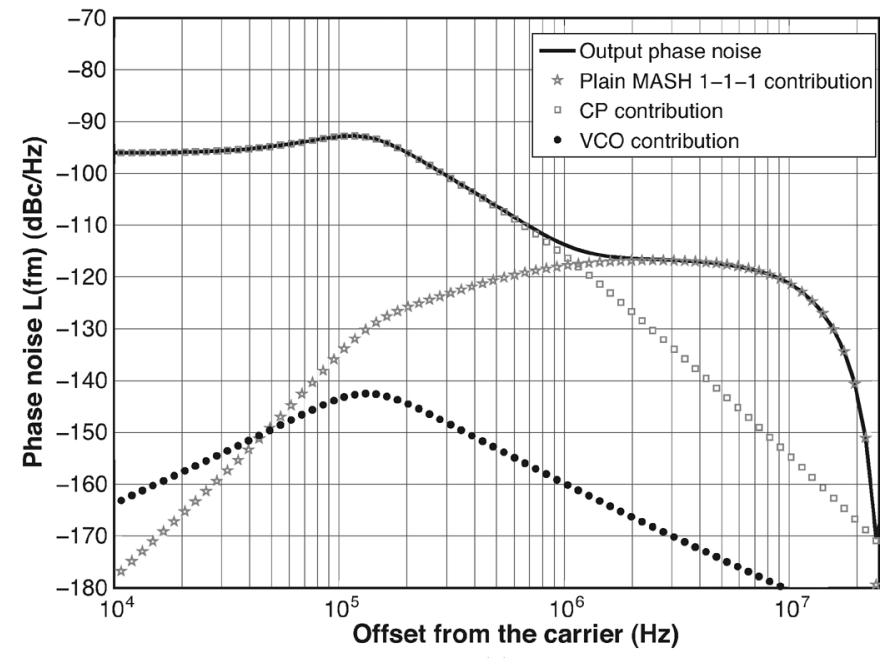

(a)

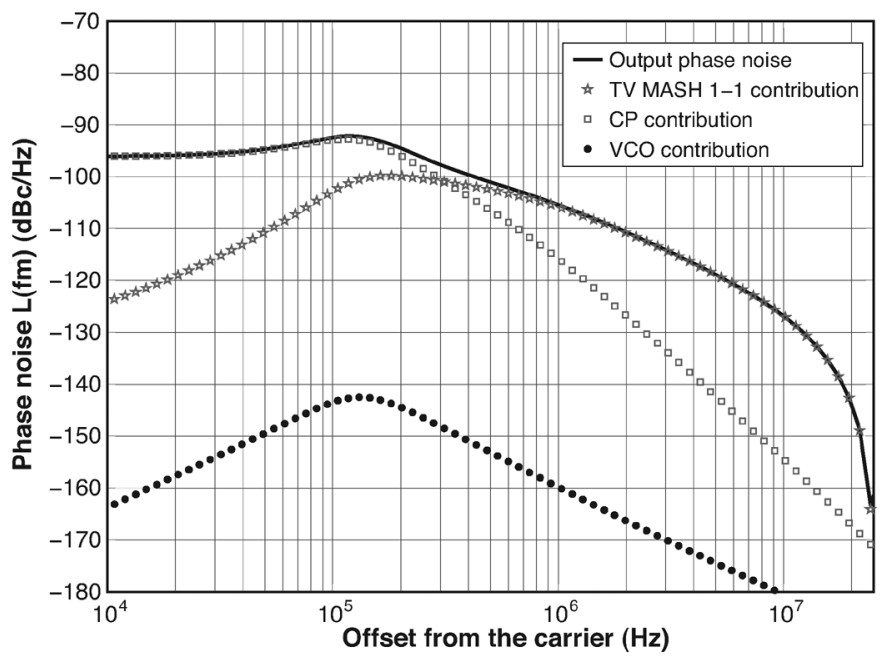

(b)

Fig. 12. Comparison of integrated phase noise. (a) Overall phase noise estimation for a MASH 1-1-1. (b) Overall phase noise estimation for the TV MASH 1-1.

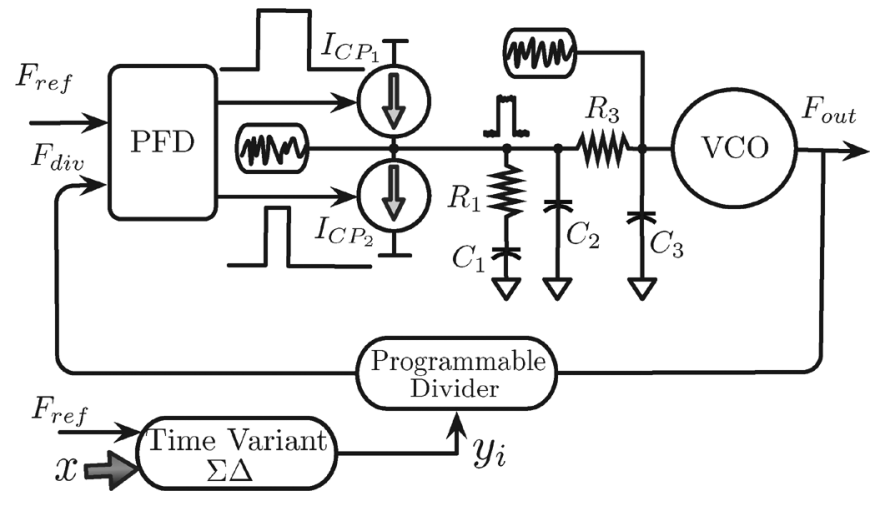

Fig. 13. Block diagram of the fractional frequency synthetizer model.

alternate known methods. Using the well know approach on [2] the frequency domain phase-noise related model is shown in Fig. 11. The overall synthesizer's output phase noise at a frequency $f_{m}$ offset from the carrier is

$$
S_{\text {synt }}\left(f_{m}\right)=\sum_{n} S_{\text {out }_{n}}\left(f_{m}\right)
$$

where $S_{\text {out }_{n}}\left(f_{m}\right)$ is the contribution from every noise source within the fractional synthesizer. Fig. 11 labels three of the major noise sources as: $S_{p d f c p}$ (from the charge pump and PFD), $S_{\text {lfvco }}$ (from the loop filter and VCO) and $S_{\Sigma \Delta}$ (from the MASH quantization). The overall phase noise at output is thus given by

$$
S_{\text {out }_{n}}\left(f_{m}\right)=\left|H_{n}\left(j 2 \pi f_{m}\right)\right|^{2} S_{\text {input }_{n}}\left(f_{m}\right) .
$$

Considering a white noise approximation, the TV MASH 1-1 $\Sigma \Delta$ quantization noise contribution is

$$
\begin{aligned}
& S_{\text {out }}\left(f_{m}\right)=\frac{1}{T_{s}}\left|T_{s} \frac{A_{f}\left(f_{m}\right)}{1+A_{f}\left(f_{m}\right)}\right|^{2}\left|2 \pi \frac{z^{-1}}{1-z^{-1}}\right|^{2} \\
& \cdot\left|\left(1-z^{-1}\right)^{m-1}+c\left(z^{-1}\left(1-z^{-1}\right)^{m-1}-z^{-1}\right)\right|^{2} S_{\Sigma \Delta}
\end{aligned}
$$

being $m$ the $\Sigma \Delta$ modulator's order. Notice that the quantization error has the same noise shaping limitation by the coefficient $c$ as predicted in (9). Therefore, the increased quantization noise

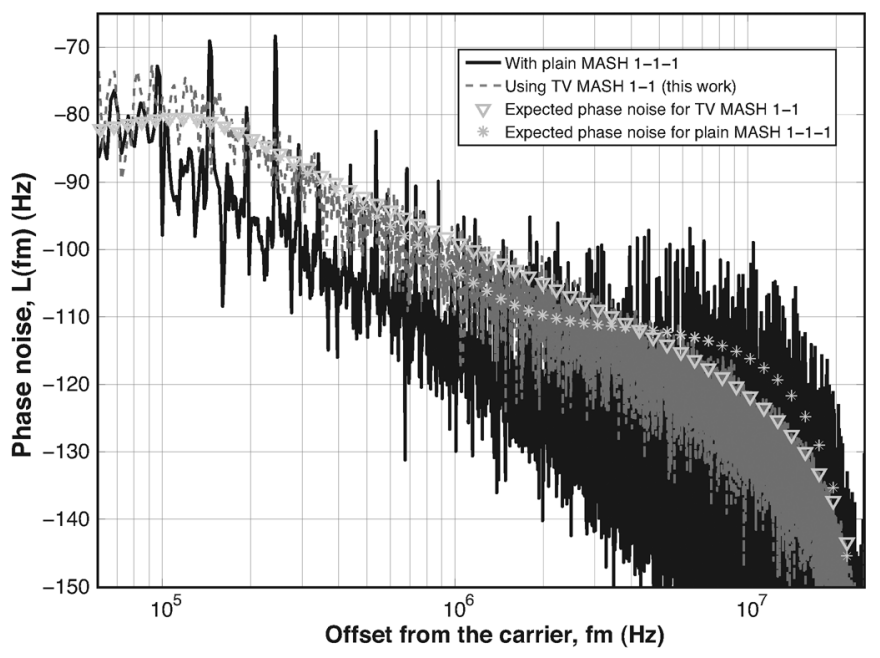

Fig. 14. Phase noise of conventional MASH 1-1-1 and time variant 1-1.

caused by the time variation of coefficients does not degrade the overall phase noise figure as clarified latter.

The synthesizer's open loop gain is

$$
A_{f}\left(f_{m}\right)=\frac{\alpha}{2 \pi} I_{c p} H_{l p f}\left(f_{m}\right) \frac{K_{V C O}}{j f_{m}} \frac{1}{N}
$$

where $H_{l p f}\left(f_{m}\right)$ is the loop's filter transfer function. To obtain the estimated phase noise for the TV MASH 1-1 we have used the parameters shown in Table II. Fig. 12(a) displays the results when (13) includes the quantization noise from a plain MASH 1-1-1 while Fig. 12(b) presents the result when for the TV MASH 1-1 [see (14)]. Simulations, consider a white noise quantization error, and predict that the TV MASH 1-1 approximation increases noise shaping for frequency ranges where phase noise is dominated by other sources such as CP and VCO noise. A higher noise shaping has lower noise at intermediate frequencies but the real limit to performances is given by the tones. Notice that the analytical study uses approximations. For instance, the charge pump mismatch is not considered in (13). The mismatch on current sources increase the phase noise for close to the carrier frequencies [3]. To include these effects in the approximation is not a trivial task. In fact it is necessary 


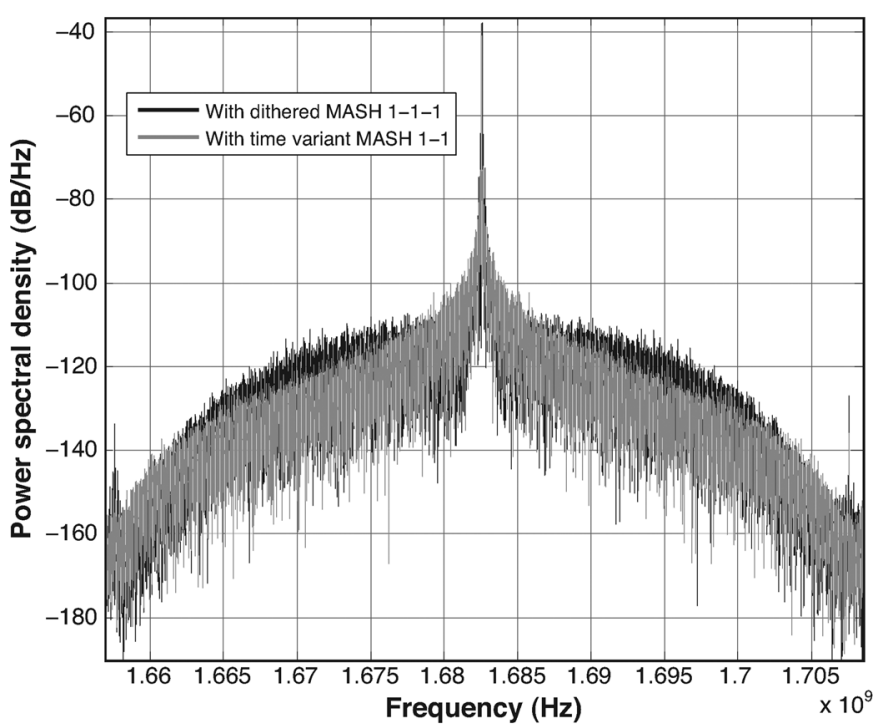

(a)

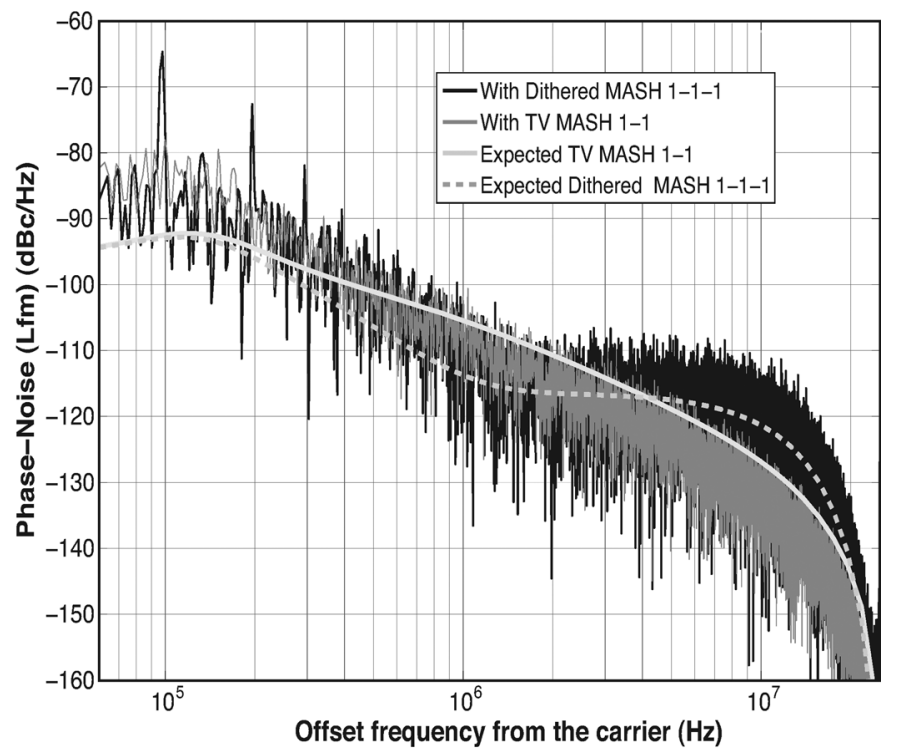

(b)

Fig. 15. Results of the verilogA simulation for a third order dithered MASH and a second order time variant MASH. (a) Power spectrum. (b) Phase noise.

an statistical analysis for the charge pump mismatch related to both the reset time in the $\mathrm{CP}$ and to the difference between phases on the PFD when the fractional synthesizer is near to "lock." For the shake of brevity we focus on the effects that bring the time variation coefficients on the $\Sigma \Delta$ modulator.

The previously mentioned non ideal effects on charge pump are included in a behavioral model in VerilogA. The model changes the value of the $\mathrm{CP}$ current sources with a proper deviation from its nominal value $I_{C P}$. The simulation let us verify that a TV MASH 1-1 exhibits a better trade off between noise shaping and number of output's quantization levels as described in the following sub section.

\section{Fractional Frequency Synthesizer Tests}

The nonlinearity around the frequency synthesizer's loop generates unpredictable intermodulation products. To estimate the effectiveness of the digital TV MASH $\Sigma \Delta$ modulator we have explored it into the synthesizer's architecture. This is done here with the verilogA model whose conception is shown in Fig. 13. The model accounts for the principal noise sources and non ideal behavior in fractional synthesizers $S_{p f d c p}\left(f_{m}\right)$, $S_{l f v c o}\left(f_{m}\right)$ and $S_{\Sigma \Delta}\left(f_{m}\right)$ [see (13), (14)]. The model uses for the charge pump mismatch (being the major limit for $\Sigma \Delta$ 's periodicity [3]) a Gaussian distribution with

$$
\begin{aligned}
& I_{C P_{1}}=I_{C P}+\Delta I_{C P_{1}} \\
& I_{C P_{2}}=I_{C P}+\Delta I_{C P_{2}}
\end{aligned}
$$

where $\Delta I_{C P_{1}}, \Delta I_{C P_{2}}$ are two random variables with Gaussian variance of $\pm 20 \%$ of the nominal $I_{C P}$ which are set up once the simulation starts. Table II resumes the remaining values of parameters: reference frequency $F_{r e f}$, the low pass filter $L P F$ order and cutoff frequency $f_{c}$, the nominal $I_{C P}$ value, the average fractional division $x / 2^{m}$ and the number of levels for which the programmable divider must change $N$.

The reference frequency is $f_{\text {ref }}=25 \mathrm{MHz}$ and the output frequency is set to $f_{\text {out }}=(67+(77 / 256)) f_{\text {ref }}=$

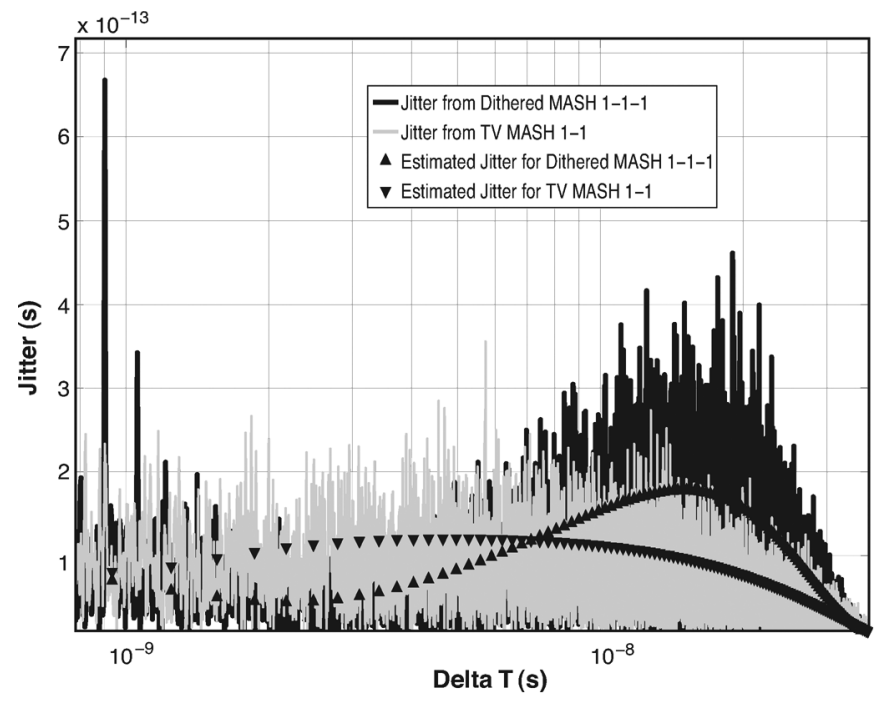

Fig. 16. Accumulated jitter for a dithered MASH 1-1-1 and the TV MASH 1-1.

1.682519 GHz. The choice is to show the benefits of the method even with nonprime values of the fractional part. A pwelch algorithm [21] applied to a $2^{22}$ size sequence estimates the power spectral density.

Fig. 14 displays the phase noise with a plain MASH 1-1-1 and the proposed TV MASH 1-1. They are close to the estimated phase noise figure in both cases but a small deviation exists for low frequencies, principally due to the inclusion of the CP mismatch in the behavioral model. The MASH 1-1-1 gives a lower noise level but the tones close to the carrier frequencies are not satisfactory. On the contrary, the time variant scheme spreads the periodicity and greatly reduces the magnitude of tones. The latter corresponds better to the expected white noise approximation from (14).

To compare the tones reduction with state of the art techniques we compared with results obtained from a dithered MASH 1-1-1 scheme. Fig. 15(a) shows the power spectral density of the simulated fractional frequency synthesizer with a 


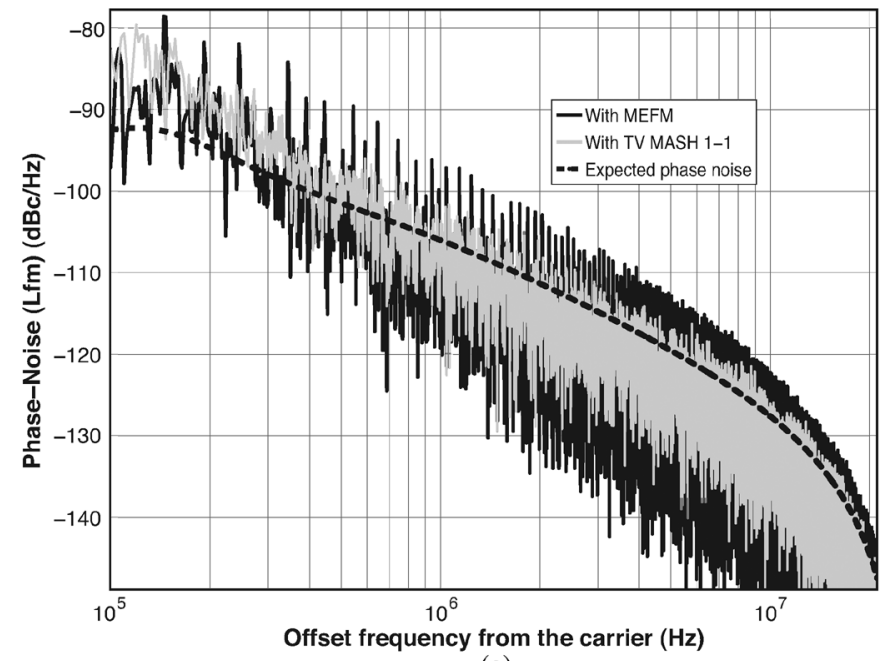

(a)

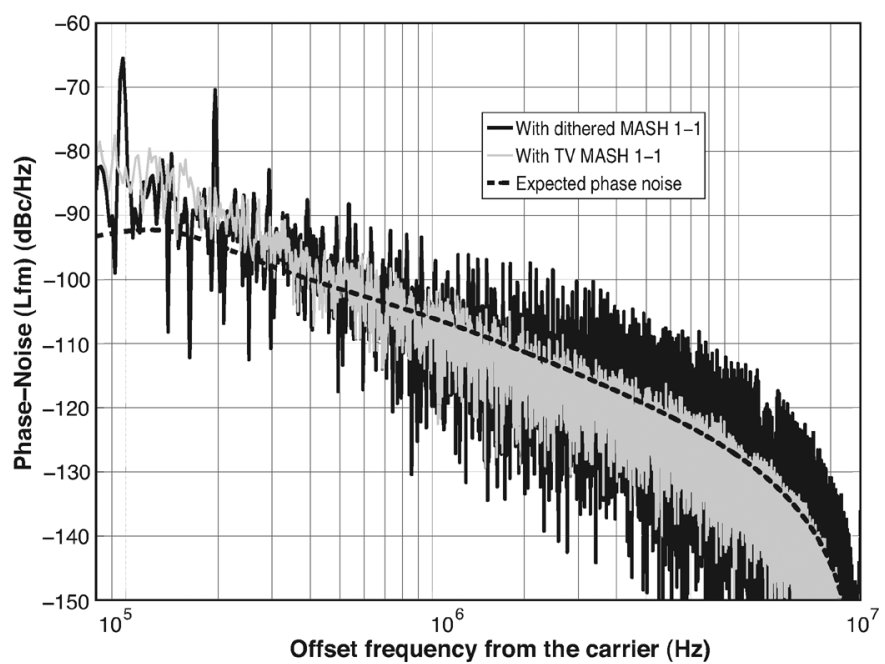

(b)

Fig. 17. Phase noise figure comparison for second order schemes. (a) Between MEFM 1-1 and TV MASH 1-1 (this work). (b) Between dithered MASH 1-1 and TV MASH 1-1 (this work).

dithered third order MASH $\Sigma \Delta$ modulator (dithered on the first accumulator) and with a second order time variant MASH $\Sigma \Delta$. It is evident the reduction of spur tones for the second order time variant topology even compared to a dithered third order scheme. The phase noise figures were obtained from those power spectrums and they are compared in Fig. 15(b). Observe that the phase noise for the dithered case presents tones for low frequencies due to the $\mathrm{CP}$ mismatch. Even the phase noise figure for intermediate frequencies is smoother for the second order time variant because the tones amplitude is considerably decreased.

It is also possible to express phase noise in time domain as jitter. Considering the approach

$$
S(f)=a\left(\frac{f_{\text {out }}}{f_{m}}\right)^{2}
$$

for an autonomous circuit such as a VCO, where $a$ is the noise power generating the time uncertainty on transitions, we can express time Jitter by $J=\sqrt{a T}$. $J$ is the square root value of the variance of jitter and $T=1 / f_{\text {out }}$ is the nominal period of the VCO [22]. However, for autonomous circuits $J_{k}=\sqrt{k a T}=$ $\sqrt{a \Delta T}$ and jitter accumulates.

Even if for a frequency synthesizer a VCO is not strictly speaking an autonomous circuit, as the phase uncertainty depends on the PLL bandwidth and nonlinearity from driven circuits within the loop. This calculus gives us another insight on the advantages of having a smooth characteristic in the TV MASH 1-1 $\Sigma \Delta$ scheme. Fig. 16 presents the average time jitter vs. $\Delta T$ obtained from the phase noise transformation of (18) and $J_{k}=\sqrt{a \Delta T}$. From the result, it becomes evident that the spur suppression is better for the TV MASH 1-1 modulator compared to a dithered MASH 1-1-1. Note we have present this result to highlight the results compared to a higher order scheme, which is supposed to avoid tones in the phase noise figure.

Considering strictly second order architectures it is important to emphasize the advantage of TV MASH 1-1 in relation to another low order schemes. Fig. 17 presents a comparison of phase noise figures obtained from these cases. For all of them, the number of levels used at the programmable divider's input is 4 (2-bit sequence) but the TV approach reduces spur tones for all cases. Fig. 17(a) shows the comparison with the MEFM 1-1 and for this case the TV approach has a smoother spectrum. Similarly, Fig. 17(b) displays the comparison with a MASH 1-1 dithered approach. The dither sequence is obtained from an 8-bit LFSR with the same characteristics as the used as the auxiliary LFSR for the TV MASH 1-1. Note the appearance of tones that evidence a strong periodicity for the dithered case. This means that the TV topology is completely different from a simple dithered scheme and gives a result a reduction of tones in the phase noise figure with the advantages mentioned along this work.

\section{CONCLUSION}

A low-order digital $\Sigma \Delta$ modulator capable to significantly reduce tones in the phase noise of a fractional frequency synthesizer has been described. It is proved, by behavioral simulations, that a pseudorandom time variation of modulator's coefficients disable the periodicity of the quantization noise better than previously known methods.

The technique requires a comparable hardware for same order modulators. The proposed TV MASH 1-1 has a smoother quantization noise shaping that allows a better trade-off between shaping and number of levels for the programmable divider. Phase noise figures are in better agreement with expected noise figures obtained with a white noise approximation, that is, prove that the TV MASH 1-1 scheme limits spur tones for fractional synthesizers. As a side benefit, a second order MASH simplifies the programable divider complexity which is one of the most stringent blocks as reliability and frequency covering range depend on the number of programmable levels.

\section{REFERENCES}

[1] T. A. D. Riley, M. A. Copeland, and T. A. Kwasniewski, "Delta-Sigma modulation in fractional-N frequency synthesis," IEEE J. Solid-State Circuits, vol. 28, no. 5, pp. 553-559, May 1993. 
[2] M. H. Perrot, M. D. Trott, and C. G. Sodini, "A modeling approach for $\Sigma \Delta$ fractional-N frequency synthesizer allowing straightforward noise analysis," IEEE J. Solid-State Circuits, vol. 37, no. 8, pp. 1028-1038, Aug. 2002.

[3] P.-E. Su and S. Parmati, "Mismatch shaping techniques to linearize charge pump errors in fractional- $N$ PLLs," IEEE Trans. Circuits Syst. I, Reg. Papers, vol. 57, no. 6, pp. 1221-1230, Jun. 2010.

[4] S. R. Norsworthy, R. Schreier, and G. C. Temes, Delta-Sigma Data Converters. New York: IEEE Press, 1997, ch. 3.

[5] Y.-H. Peng and L.-H. Lu, "A 16-GHz triple-modulus phase-switching prescaler and its application to a $15-\mathrm{GHz}$ frequency synthesizer in 0.18 $\mu \mathrm{m}$ CMOS," IEEE Trans. Microw. Theory Tech., vol. 55, no. 1, pp. 44-51, Jan. 2007.

[6] A. M. Fahim and M. I. Elmasry, "A wideband Sigma-Delta phaselocked-loop modulator for wireless applications," IEEE Trans. Circuits Syst. II, Analog Digit. Signal Process., vol. 50, no. 2, pp. 53-62, Feb. 2003.

[7] K. Hosseini and M. P. Kennedy, "Mathematical analysis of a prime modulus quantizer MASH digital Delta-Sigma modulator," IEEE Trans. Circuits Syst. II, Exp. Briefs, vol. 54, no. 12, pp. 1105-1109, Dec. 2007.

[8] K. Hosseini and M. P. Kennedy, "Maximum sequence length MASH digital Delta-Sigma modulators," IEEE Trans. Circuits Syst. I, Reg. Papers, vol. 54, no. 12, pp. 2628-2638, Dec. 2007.

[9] K. Hosseini and M. P. Kennedy, "Architectures for maximum-sequence-length digital Delta-Sigma modulators," IEEE Trans. Circuits Syst. II, Exp. Briefs, vol. 55, no. 11, pp. 1104-1108, Nov. 2008.

[10] M. J. Borkowski and J. Kostamovaara, "Post modulador filtering in $\Sigma \Delta$ fractional-N frequency synthesis," in Proc. IEEE Int. Midwest Symp. Circuits Syst. (MWSCAS), Hiroshima, Japan, Jul. 2004, pp. $\mathrm{I}-325-\mathrm{I}-328$.

[11] M. J. Borkowski and J. Kostamovaara, "On randomization of digital Delta-Sigma modulators with DC inputs," in Proc. IEEE 2006 Int. Symp. Circuits Syst. (ISCAS), May 2006, pp. 3770-3773.

[12] Y.-H. Liu and T.-H. Lin, "A wideband PLL-based G/FSK transmitter in $0.18 \mu \mathrm{m}$ CMOS," IEEE J. Solid-State Circuits, vol. 44, no. 9, pp. 2452-2462, Sep. 2009.

[13] S. Parmati, J. Welz, and I. Galton, "Statistics of the quantization noise in 1-Bit dithered single-quantizer digital Sigma-Delta modulators," IEEE Trans. Circuits Syst. I, Reg. Papers, vol. 54, no. 3, pp. 492-503, Mar. 2007.

[14] V. R. Gonzalez-Diaz, M. A. Garcia-Andrade, G. E. Flores-Verdad, and F. Maloberti, "Efficient dithering in MASH Sigma-Delta modulators for fractional frequency synthesizers," IEEE Trans. Circuits Syst. I, Reg. Papers, vol. 57, no. 9, pp. 2394-2403, Sep. 2010.

[15] F. Maloberti, Data Converters. Dordrecht, The Netherlands: Springer, 2008, ch. 1

[16] E. A. Lee and D. G. Messerschmitt, Digital Communication. Boston, MA: Kluwer Academic, 1994, ch. 12.

[17] K. Shu, E. Sánchez-Sinencio, J. Silva-Martínez, and S. H. K. Embabi, "A $2.4 \mathrm{GHz}$ monolithic fractional-N frequency synthesizer with robust phase-switching prescaler and loop capacitance multiplier," IEEE $J$. Solid-State Circuits, vol. 38, no. 6, pp. 866-873, Jun. 2003.

[18] J. Liu, H. Liao, R. Huang, and X. Zhang, "Sigma-Delta modulator with feedback dithering for RF fractional-N frequency synthesizer," in Proc. IEEE Conf. Electron Devices Solid-State Circuits, Dec. 2005, pp. 137-139.

[19] B. D. Muer and M. Steyaert, CMOS Fractional-N Synthesizers. Boston, MA: Kluwer Academic, 2003.

[20] Y.-H. L. , Y.-C. Yang, S.-A. Yu, and S.-S. Lu, "A quantization noise suppression technique for $\Sigma \Delta$ fractional- $N$ frequency synthesizers," IEEE J. Solid-State Circuits, vol. 41, no. 11, pp. 2500-2511, Nov. 2006.
[21] J. G. Proakis and D. G. Manolakis, Digital Signal Processing: Principles, Algorithms and Applications. Upper Saddle River, NJ: Prentice-Hall, 1996, ch. 12.

[22] K. S. Kundert, "Introduction to RF simulation and its application," IEEE J. Solid-State Circuits, vol. 34, no. 9, pp. 1298-1319, Sep. 1999.

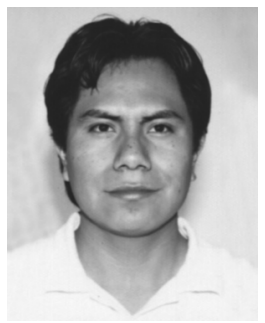

Victor Rodolfo Gonzalez-Diaz was born in Puebla, Mexico. He received the B.Sc. degree on electronics engineering from the Technological Institute of Puebla in 2003 and the M.Sc. and Ph.D. degrees from the National Institute for Astrophysics, Optics and Electronics (INAOE), Puebla, in 2005 and 2009, respectively.

He collaborated as a Postdoctoral Fellow at the Microsystems Laboratory, University of Pavia, Italy, from 2009 to 2010 . He is currently a full time Researcher with BUAP Electronics Faculty, Puebla. His main research interests are frequency synthesizers, data converters, and Sigma-Delta modulation for analog and digital applications.

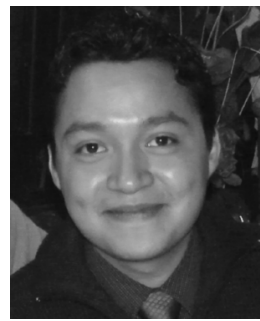

Aldo Pena-Perez was born in Queretaro, Mexico, in 1981. He received the B.Sc. degree in electronics engineering from the Technological Institute of Queretaro in 2004 and the M.Sc. degree from the National Institute for Astrophysics, Optics and Electronics (INAOE) Puebla, Mexico, in 2006. He pursued the Ph.D. degree from the Microsystems Laboratory of University of Pavia, Italy, in 2010 During his Ph.D., he worked on development, design and testing of low-power sigma delta modulators.

$\mathrm{He}$ is currently a Postdoctoral Fellow at the Microsystems Laboratory of University of Pavia. His main research interests are focused on mixed signal design, low-voltage, low-power oversampled data converters, and analog amplifiers design.

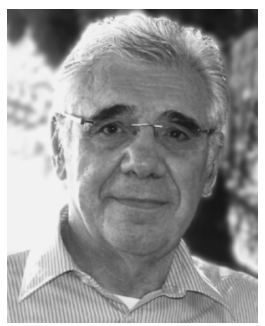

Franco Maloberti received the Laurea degree in physics (summa cum laude) from the University of Parma, Italy, in 1968, and the Doctorate Honoris Causa degree in electronics from the Instituto $\mathrm{Na}$ cional de Astrofisica, Optica y Electronica (INAOE), Puebla, Mexico, in 1996.

He was a Visiting Professor at the Swiss Federal Institute of Technology (ETH-PEL), Zurich, and at the EPFL, Lausanne, Switzerland. He was the TI/J. Kilby Chair Professor at Texas A\&M University, and the Distinguished Microelectronic Chair Professor a the University of Texas at Dallas. Presently he is Professor of Microelectronics and Head of the Micro Integrated Systems Group, University of Pavia, Italy.

Dr. Maloberti was the recipient of the XII Pedriali Prize for his technical and scientific contributions to national industrial production, in 1992. He was corecipient of the 1996 Institute of Electrical Engineers Fleming Premium, the best paper award, ESSCIR-2007, and the best paper award, IEEJ Analog Workshop-2007 and 2010. He received the 1999 CAS Society Meritorioius Service Award, the 2000 CAS Society Golden Jubilee Medal, and the 2000 IEEE Millennium Medal. 